\title{
ECMO Therapy in Acute Chest Syndrome for Patients with Sickle Cell Disease: a Case Report and Literature Review
}

\author{
Soi Avgeridou ${ }^{1}$ (D) Ilija Djordjevic ${ }^{1} \cdot$ Anton Sabashnikov $^{1} \cdot$ Kaveh Eghbalzadeh $^{1} \cdot$ Laura Suhr $^{1} \cdot$ Borko Ivanov $^{1}$. \\ Julia Merkle ${ }^{1} \cdot$ Christian Rustenbach $^{1} \cdot$ Navid Mader $^{1} \cdot$ Axel Kröner $^{1} \cdot$ Thorsten Wahlers $^{1}$
}

Accepted: 1 June 2021 / Published online: 3 July 2021

(C) The Author(s) 2021

\begin{abstract}
Extracorporeal membrane oxygenation (ECMO) plays an important role as a life-saving tool for patients with therapy-refractory cardio-respiratory failure. Especially, for rare and infrequent indications, scientific data is scarce. The conducted paper focuses primarily on our institutional experience with a 19-year-old patient suffering an acute chest syndrome, a pathognomonic pulmonary condition presented by patients with sickle cell disease. After implementation of awake ECMO therapy, the patient was successfully weaned off support and discharged home 22 days after initiation of the extracorporeal circulation. In addition to limited data and current literature, further and larger data sets are necessary to determine the outcome after ECMO therapy for this rare indication.
\end{abstract}

Keywords Sickle cell disease $\cdot$ Awake ECMO $\cdot$ Respiratory failure

\section{Introduction}

Sickle cell disease (SCD) is an autosomal recessive hemoglobinopathy leading to the formation of rigid sickled-shaped red blood cells due to polymerization of hemoglobin [1]. Next to various complications, pulmonary embolism (PE) is reported to be associated with a $6 \%$ mortality rate among SCD patients in a retrospective case-control study performed by Novelli and colleagues [2]. Severe forms of PE can trigger hemodynamic deterioration, as well as contribute to an acute chest syndrome (ACS), pathognomonic for SCD patients [2]. Clinical data showed an underestimation of PE in SCD patients [2]. However, the detection of SCD in patients with acute PE without any prior history of SCD might be challenging. Furthermore, avoidance of concomitant complications should aim early diagnostics and prompt therapy.

Soi Avgeridou and Ilija Djordjevic contributed equally to this work.

This article is part of the Topical Collection on Surgery

Soi Avgeridou

soi.avgeridou@uk-koeln.de

1 Department of Cardiothoracic Surgery, Heart Center Cologne, University Hospital Cologne, Kerpener Str. 62,

50937 Cologne, Germany
In this regard, we report a case of a young patient with a primary presentation of an acute PE and concomitant ACS, treated with venoarterial extracorporeal membrane oxygenation (VA-ECMO) due to severe cardio-respiratory failure. Furthermore, we present a review of current literature concerning this complex and specific patient cohort.

\section{Report}

A 19-year-old patient with African roots was initially admitted to a secondary care hospital due to severe dyspnea and tachycardia. Echocardiographic diagnostics showed a massive impairment of right heart function and led to detection of a $\mathrm{PE}$ in the conducted computed tomography (CT). Thrombolytic therapy with alteplase was initiated and the patient showed subjective improvement. Nevertheless, in a later CT-scan, the PE was still evident. The presence of a deep vein thrombosis was not verifiable. In an acceptable cardio-respiratory state, rivaroxaban was established as further anticoagulation treatment. However, 2 days later, the patient developed fever, a rise of inflammation parameters, as well as a progress of pneumonic infiltrates in the CT scan (Fig. 1). Accordingly, antibiotics (Piperacillin/Tazobactam) were implemented.

Six days after initial treatment, the patient showed signs of re-exacerbation of initial pulmonary and cardiac organ 


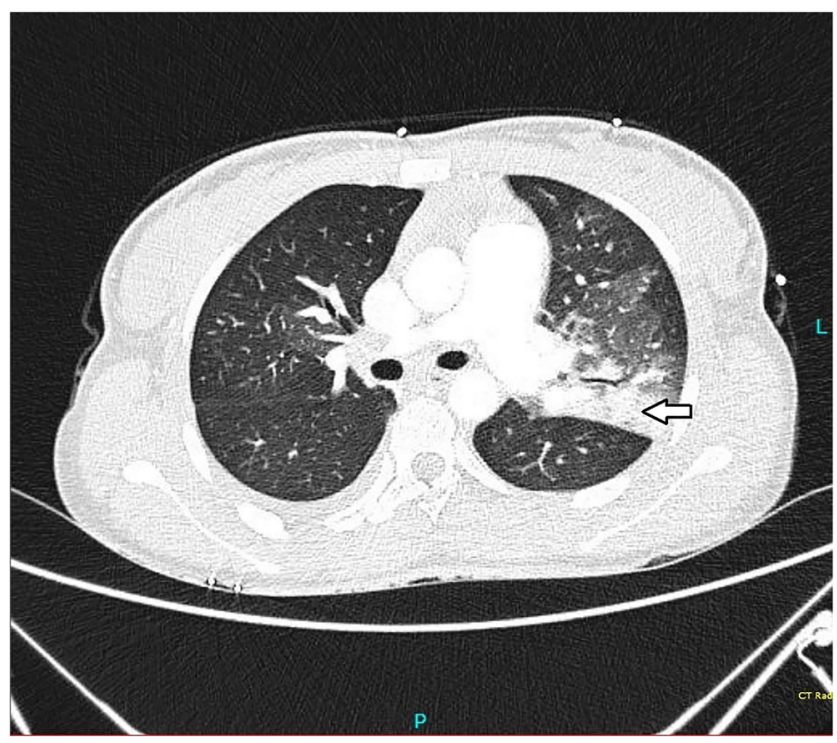

Fig. 1 Computed tomography showing pneumonic infiltrates in the left upper lobe of the lung

dysfunction. The chest $\mathrm{CT}$ scan promptly performed revealed a central presentation of a PE (Fig. 2).

Echocardiography showed severe signs of right ventricular failure. Additionally, severe hypoxia developed despite the attempt of non-invasive ventilation with $100 \% \mathrm{~F}_{\mathrm{i}} \mathrm{O}_{2}$, which in turn led to a fulminant hemodynamic decline and excessive need for vasoconstrictive therapy. Arterial blood gas analysis showed the following parameters: $\mathrm{SO}_{2}, 58.8 \% ; \mathrm{PO}_{2}, 33$; $\mathrm{PCO}_{2}, 29 ; \mathrm{BE}, 6.6 ; \mathrm{HCO}_{2}, 17.0 ; \mathrm{Hb}, 10.1 ; \mathrm{pH}, 7.38 ; \mathrm{Na}^{+}$, 143; $\mathrm{K}^{+}, 3.3$; Lactate, 2.9 ; and $\mathrm{Ca}^{2+}, 1.24$.

Therefore, the patient was admitted to our department for further treatment in this therapy-refractory state of cardiopulmonary deterioration.

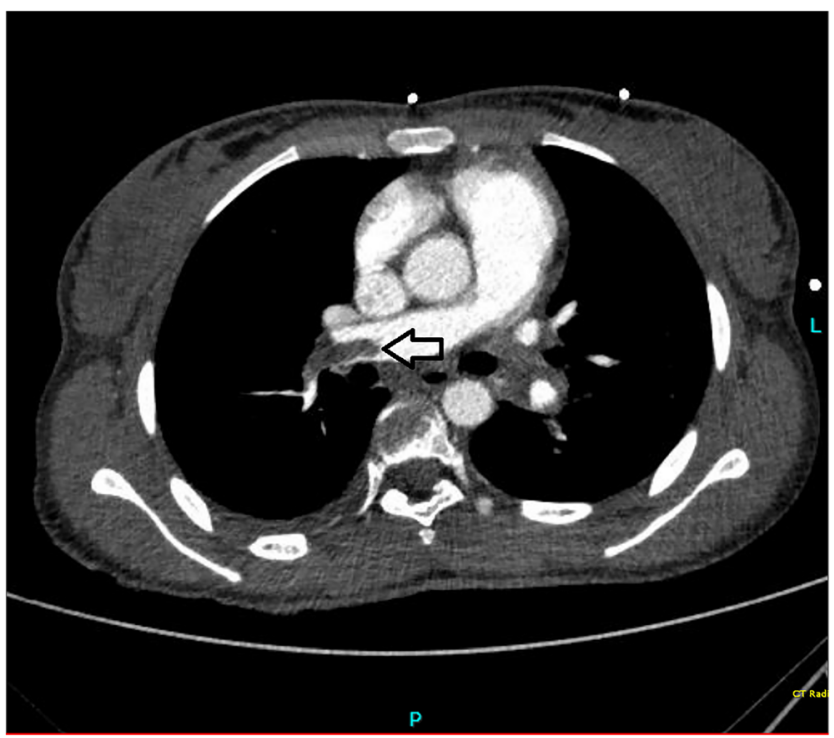

Fig. 2 Computed tomography showing pulmonary embolus
Due to initial failure of the treatment with alteplase, repetition of thrombolysis therapy was not performed. Moreover, surgical extraction of the thrombus formation was not considered beneficial due to high risk of intra- and postoperative bleeding. The initiation of mechanical ventilation seemed to be unavoidable. However, we decided to implement an awake VA-ECMO therapy via inguinal vessels (Rotaflow, Maquet, Rastatt, Germany), to avoid further hemodynamic deterioration due to the accompanying necessity of analgosedation. Initial ECMO parameters were $\mathrm{FiO}_{2} 100 \%$, gas-flow $3.0 \mathrm{~L} /$ min, and ECMO-flow $3.90 \mathrm{~L} / \mathrm{min}$.

Under ECMO support, an angiography-driven thrombectomy was performed with improvement of hemodynamics and respiratory state in the following days. The application of unfractionated heparin targeting an activated clotting time of 180 to $200 \mathrm{~s}$ resulted in heparin-induced thrombocytopenia necessitating therapy with argatroban. Fortunately, there was no tendency of clot formation with the administered anticoagulation. Particularly, lung recovery was noticeable in the following chest-X-rays (Fig. 3). When ECMO weaning was initiated, a nasal high flow was established to ensure a successful transition. During this time, the patient was limited to mobilization inside the bed. Six days after initiation of ECMO circulation, the patient could be weaned off support.

However, short-term follow-up diagnostics could exclude a paraneoplastic genesis of PE. Moreover, the conducted blood smear showed a hypogranulation of neutrophil granulocytes. The share of lymphocytes was within a normal range. Due to increased platelets (thrombocytes: $505 \times 1^{9} / \mathrm{L}$ ) on admission, further testing in the direction of genetic predisposition of thrombotic events showed a factor $\mathrm{V}$ disorder of the normal genotype. Furthermore, hemoglobin

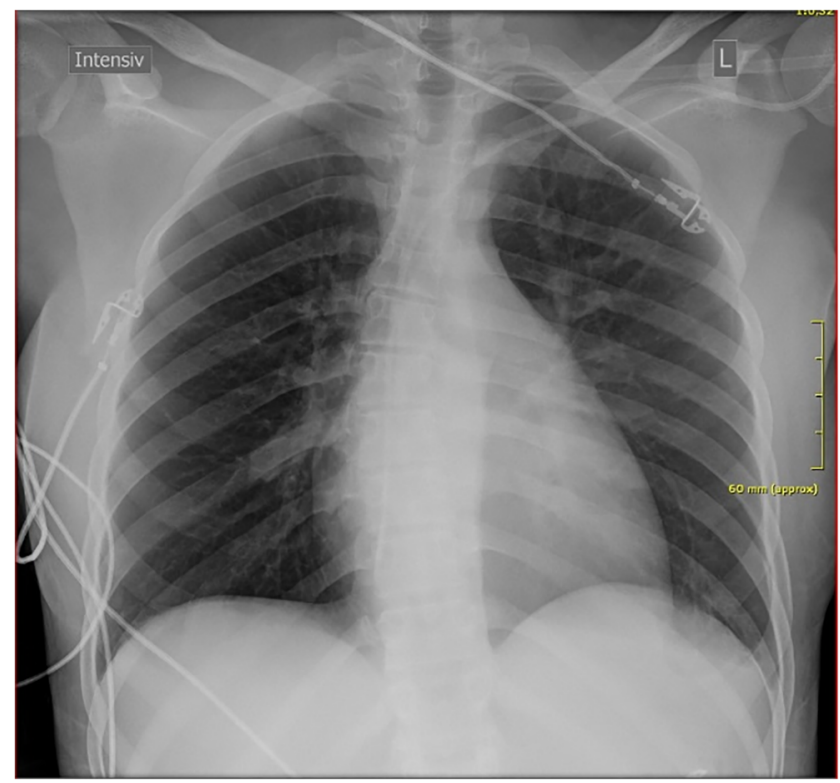

Fig. 3 X-ray of the chest 3 days after ECMO explantation showing no signs of pathology after treatment 
electrophoresis clearly showed homozygous sickle cell hemoglobinopathy (hemoglobin S 49.8\%, hemoglobin F 48.2\%, hemoglobin A 20\%). Additionally, the patient's father suffered from SCD as he reported at a later point. Based on the diagnosis of SCD, anticoagulation treatment was implemented with phenprocoumon targeting an international normalized ratio (INR) of 2.5 to 3.5. The range was set at a higher target than usual, given the presented risk factors.

Without any further disturbances following an uneventful course on the normal ward, the patient was discharged 22 days after ECMO therapy. In a 5-month follow-up, the patient showed no signs of remaining cardio-respiratory failure, no thrombotic events, and no exacerbation of SCD.

\section{Discussion and Literature Review}

ECMO therapy is widely used as salvage therapy in patients developing therapy refractory PE [3]. However, we describe the first case of an awake VA-ECMO therapy due to PE and following ACS in a patient with unknown SCD.

Next to various complications concerning vaso-occlusive conditions associated with SCD, PE and ACS are serious complications and common reasons for hospitalization as well as death in patients suffering from SCD [2]. In this regard, ECMO circulation may serve as a life-saving treatment option for this rare patient cohort.

\section{Veno-Venous (VV)-ECMO Therapy in Patients with ACS}

Few reports are available in the literature concerning SCD patients with need for VV-ECMO therapy. Moreover, the majority of reports presented patients with initial ACS and already diagnosed SCD. Sewaralthahab and colleagues reported the first adult patient in the USA suffering from ACS and being treated with VV-ECMO. After 20 days of ECMO treatment and full recovery of respiratory function, the patient was weaned off support and transferred to the referring hospital [4]. Mashhour et al. presented a case of a 14-year-old child with SCD and therapy refractory ACS, necessitating ECMO therapy. The ECMO was explanted $111 \mathrm{~h}$ after initiation, and the patient was discharged from the hospital 1 month after admission. Finally, the authors conclude ECMO therapy in SCD patients to be associated with increasing survival compared to conservative treatment with mechanical ventilation and medical therapy [5].

In a report from Germany, a 28-year-old patient developed a vaso-occlusive crisis due to massive alcohol consumption. Upon admission, the patient was intubated and severe respiratory failure indicated VV-ECMO therapy. After successful treatment, the patient left the hospital 19 days after the emergency hospitalization [6].
Another case of a young patient with known SCD was described by Parhar and colleagues. Due to food poisoning, emergent hospitalization was necessary indicating the establishment of mechanical ventilation due to hypoxemic respiratory failure and hemodynamic deterioration. Further diagnostics showed a bilateral lung consolidation. Even though the treatment was escalated with exchange transfusions, ongoing pulmonary failure led to an implantation of VV-ECMO via a femoro-femoral access. ECMO therapy was successfully finished after 12 days. Unfortunately, a follow-up after 6 months presented chronic respiratory insufficiency. Despite of this, the authors suggested a femoro-femoral approach to be associated with lower risk of stroke on ECMO. Moreover, they described the use of a second oxygenator in case of insufficient $\mathrm{CO}_{2}$-clearance [1].

The earliest paper describing VV-ECMO as a treatment option for sickle cell disease was published in 1997. The report described a case of an 8-year-old boy undergoing a vasoocclusive crisis, quickly progressing into an acute chest syndrome. Due to therapy-refractory pulmonary insufficiency, VV-ECMO therapy was established. After 11 days, a successful weaning process was conducted. In a 5-year follow-up, the patient showed no residue of abnormal pulmonary function [7]. In the report of Al-Sawaf et al., the authors described a 21year-old patient with homozygote SCD being transferred from another hospital due to acute respiratory failure. After being treated with fluids and intravenous opioids due to severe pain, he developed purulent sputum and a worsened general state. VV-ECMO therapy was established in fulminant respiratory failure. After 7 days, the patient could be weaned off support. The patient was discharged from the hospital 4 weeks upon admission. In their report, the authors specifically addressed the need for sufficient hemoglobin levels during ECMOtherapy in SCD-patients achieved through exchange transfusions [8]. Concerning our case, exchange transfusions were not necessary in regard of acceptable hemoglobin levels.

\section{VA-ECMO Therapy in Patients with ACS and Hemodynamic Decline}

In contrast to most of the reports mentioned, we described the use of VA-ECMO support due to hemodynamic and concomitant respiratory failure. The report by Quentin et al. focusses also on VA-ECMO support. However, they treated a patient with postcardiotomy cardiogenic shock and known SCD pathology prior to surgery, making in this way a comparison to our case description difficult [9].

Similar to our experience, Grein et al. presented a patient with fulminant PE, lysis therapy, and initiation of VA-ECMO circulation. Moreover, the patient had a medical history consisting of two prior events of ACS in his childhood. Finally, ECMO therapy was finished 6 days after and the patient was successfully discharged home [10]. 


\section{Studies Analyzing ECMO Therapy in SCD Patients}

What case reports mentioned combine is that they highlight the positive aspects of implemented ECMO treatment and their impact on patients with SCD and ACS. Beside the mentioned case reports, there are only two original studies with special attention on patients with ECMO support due to ACS and their outcome (Table 1). The multicenter study by Boissier et al. investigated 22 patients of which $45 \%$ ( $n=$ 10) were treated with VV-ECMO and $55 \%(n=12)$ with VA-ECMO. The authors showed a significant impact of the initial cardio-respiratory state prior to ECMO therapy on the outcome of examined patients [11]. The second study studied all SCD patients listed in the ELSO-registry from 1990 to 2012 [12]. Sixty-five patients with an age range from 30 days to 18 years were analyzed in this report. Even though respiratory failure was the most common indication for ECMO-treatment, $80 \%$ of the patients were treated with VA-ECMO. The median ventilation time of pre-ECMO mechanical ventilation was $15.5 \mathrm{~h}$ (range: 0-859 h), while median duration of ECMO treatment was $155 \mathrm{~h}$ (range: 5-689 h). The most common complications were kidney injury (45\%), bleeding (25\%), inotrope treatment $(40 \%)$, and oxygenator clotting (15\%). The overall survival was $52 \%$. The VV group showed a higher survival with $85 \%$ of cases compared to only $43 \%$ in the VA group [12].

\section{Awake ECMO Therapy-New Approach for SCD Patients}

The implementation of awake ECMO therapy has gained popularity since it poses a viable alternative with clear benefits in patients with severe respiratory failure while avoiding negative effects associated with mechanical ventilation. A review from 2016 shed some more light at the pathophysiology and clinical considerations for the use of awake ECMO [13]. The authors highlighted the physiological movement of the dorsal diaphragm, resulting in the ventilation of more compliant parts of the lung and therefore creating optimal ventilation, as the main benefits. Also, spontaneously breathing patients use their respiratory muscle, which in turn ensures the expansion of the chest wall, again leading to favorable ventilation and minimized risk of developing atelectasis. Through spontaneous breathing, the air reaches the alveoli through a decrease of intrathoracic pressure, having no effect on the cardiac filling and output. In contrast, mechanical ventilation increases the intrathoracic pressure during inspiration, which in turn lowers the cardiac output [13].

Mohite and colleagues from Royal Brompton Hospital Harefield London analyzed ECMO patients bridged to lung transplantation and paid special attention to those supported and kept awake (7 out of 249). They claimed the clear benefit of avoiding sedation and accompanied immobilization and mechanical ventilation, without any significant difference being detected in the 1-year survival rate between the cohorts [14]. In addition, Tudorache and colleagues showed for the early phase after lung transplantation clear ameliorative impact on hemodynamics in patients bridged with awake ECMO [15].

At this point, it is of utter importance to mention this form of treatment can only be performed in patients with the highest form of compliance. In our case, there was a well-established form of communication between the patient and treating professionals ensuring the safety, as well as the comfort of the patient.

Next to promising results concerning awake ECMO therapy, there are limited data investigating this form of therapy in SCD patients. Concomitant to our report, colleagues from France implanted an awake VV-ECMO in a patient with severe $\mathrm{ACS}$ and threatening right heart failure. Maintaining

Table 1 Overview of all ECMO studies in sickle cells patients

\begin{tabular}{|c|c|c|c|c|c|c|c|c|}
\hline Study & Author & Year of publication & $\mathrm{n}$ & Age (years) & Sickle cell disease & ECMO type & ECMO duration (days) & In hospital Survival \\
\hline 1 & K. Parhar & 2015 & 1 & 18 & Yes & VV & 12 & Yes \\
\hline 2 & T. Belveyre & 2019 & 1 & 19 & Yes & VV & 7 & Yes \\
\hline 4 & S.S. Sewaralthahab & 2018 & 1 & 25 & Yes & VV & 20 & \\
\hline 5 & A. Mashhour & 2017 & 1 & 14 & Yes & VV & 5 & Yes \\
\hline 6 & M. Hoffmann & 2011 & 1 & 28 & Yes & VV & 12 & Yes \\
\hline 7 & M. A. Pelidis & 1997 & 1 & 8 & Yes & VV & 11 & Yes \\
\hline 8 & O. Al-Sawaf & 2019 & 1 & 21 & Yes & VV & 7 & Yes \\
\hline 9 & Q. de Roux & 2019 & 1 & 35 & Yes & VA & 6 & Yes \\
\hline 10 & E. Grein & 2012 & 1 & 21 & Yes & VA/VV & 11 & Yes \\
\hline 11 & F. Boissier & 2018 & 22 & $27 *[21-1]$ & Yes & VA/VV & $6^{*}[2-8]$ & $27 \%$ \\
\hline $12 * *$ & K. Kuo & 2015 & 65 & $7 *[1 \mathrm{~m}-17]$ & Yes & VA/VV & $6^{*}[5 \mathrm{~h}-28]$ & $52 \%$ \\
\hline
\end{tabular}

$A C S$ acute chest syndrome; ECMO extracorporeal membrane oxygenation; $V V$ veno-venous; $V A$ veno-arterial

*Median, [range] **Kuo et al. include a case report as well as the data from the ELSO registry 
spontaneous breathing, the patient improved and was weaned off ECMO support 7 days later [2]. Particularly, our cases are the first to describe awake ECMO therapy in this rare patient cohort with successful results for urgent onset of symptoms.

\section{Conclusion}

Current literature concerning patients suffering ACS and treated with ECMO is limited to a few case reports and two original studies with low numbers of patients. However, acceptable results for this rare patient cohort are presented. In therapy-refractory cardio-respiratory failure, ECMO therapy should be considered a potential treatment option for patients with SCD. Moreover, adequate treatment should always be administered with simultaneous diagnostics to fully address genesis of cardio-respiratory failure, especially in patients without prior history of SCD as it is described in our case. In this regard, interdisciplinary treatment and deliberation are certainly of paramount importance.

In addition, spontaneous breathing during ECMO support may avoid adverse effects of mechanical ventilation and therefore should be considered a contemporary therapeutic approach, also for patients with scarce indications.

In conclusion, further and larger data sets are needed to determine outcome after ECMO therapy in this special patient cohort.

Author Contribution Ilija Djordjevic and Soi Avgeridou were responsible for the concept design and writing. The literature review was conducted by Laura Suhr, Borko Ivanov, Stephen Gerfer, and Julia Merkle. Kaveh Eghbalzadeh played roll in the data analysis, as well as interpretation. Christian Rustenbach, Navid Mader, Axel Kröner, and Anton Sabshnikov reviewed the case report and conducted to initial treatment of the patient. Thorsten Wahlers supported the case report by providing materials and expertise.

Funding Open Access funding enabled and organized by Projekt DEAL.

Data Availability Any further data and material can be received through contacting the corresponding author.

Code Availability Not applicable.

\section{Declarations}

Ethics Approval The ethics committee simply required the written statement of consent by the patient.

Consent to Participate There is written consent of participation by the patient.

Consent for Publication There is written consent of publication by the patient.
Competing Interests There are no conflicts of interest for any of the authors.

Open Access This article is licensed under a Creative Commons Attribution 4.0 International License, which permits use, sharing, adaptation, distribution and reproduction in any medium or format, as long as you give appropriate credit to the original author(s) and the source, provide a link to the Creative Commons licence, and indicate if changes were made. The images or other third party material in this article are included in the article's Creative Commons licence, unless indicated otherwise in a credit line to the material. If material is not included in the article's Creative Commons licence and your intended use is not permitted by statutory regulation or exceeds the permitted use, you will need to obtain permission directly from the copyright holder. To view a copy of this licence, visit http://creativecommons.org/licenses/by/4.0/.

\section{References}

1. Parhar K, Parizkova B, Jones N, Valchanov K, Fowles JA, Besser $\mathrm{M}$, et al. Extracorporeal membrane oxygenation for the treatment of adult sickle cell acute chest syndrome. Perfusion. Apr. 2016;31(3): 262-5. https://doi.org/10.1177/0267659115593172.

2. Belveyre T, Auchet T, Levy B. Spontaneous breathing during extracorporeal membrane oxygenation treatment of sickle cell disease acute chest syndrome. Respir Med Case Rep. 2019;28:100924. https://doi.org/10.1016/j.rmcr.2019.100924.

3. Al-Bawardy R, et al. Extracorporeal membrane oxygenation in acute massive pulmonary embolism: a case series and review of the literature. Perfusion. Jan. 2019;34(1):22-8. https://doi.org/10. $1177 / 0267659118786830$.

4. Sewaralthahab SS, Menaker J, Law JY. Successful use of venovenous extracorporeal membrane oxygenation in an adult patient with sickle cell anemia and severe acute chest syndrome. Hemoglobin. Jan. 2018;42(1):65-7. https://doi.org/10.1080/ 03630269.2018 .1450755 .

5. Mashhour A, Easo J, Horst M, Weyland A, Zundel J, Eichstaedt $\mathrm{HC}$, et al. Extracorporeal lung support in acute chest syndrome associated with sickle cell disease: a rare report of a common case. Artif Organs. Jul. 2017;41(7):688-9. https://doi.org/10.1111/aor. 12935.

6. Hoffmann M, Geldner G, Leschke M. Lebensbedrohliches akutes Thoraxsyndrom mit hämolytischer Krise bei Sichelzellerkrankung. DMW - Dtsch Med Wochenschr. Oct. 2011;136(43):2192-5. https://doi.org/10.1055/s-0031-1292031.

7. Pelidis MA, Kato GJ, Resar LMS, Dover GJ, Nichols DG, Walker LK, et al. Successful treatment of life-threatening acute chest syndrome of sickle cell disease with venovenous extracorporeal membrane oxygenation. J Pediatr Hematol Oncol. Oct. 1997;19(5):45961. https://doi.org/10.1097/00043426-199709000-00010.

8. Al-Sawaf O, Köhler P, Eichenauer DA, Böll B, Kochanek M, Shimabukuro-Vornhagen A. Management of an adult patient with sickle cell disease and acute chest syndrome by veno-venous extracorporeal membrane oxygenation. Ann Hematol. Mar. 2019;98(3): 789-91. https://doi.org/10.1007/s00277-019-03596-z.

9. Q. de Roux et al., "Veno-arterial ECMO in sickle cell disease for urgent cardiac surgery," Ann Thorac Surg, Jul. 2019, https://doi. org/10.1016/j.athoracsur.2019.05.093.

10. Grein E, Ducrocq N, Kimmoun A, Vanhuyse F, Gerard A, Levy B. Syndrome thoracique aigu chez l'adulte drépanocytaire : intérêt de l'ECMO. Ann Fr Anesth Réanimation. Dec. 2012;31(12):973-5. https://doi.org/10.1016/j.annfar.2012.10.002.

11. Boissier F, et al. Extracorporeal life support for severe acute chest syndrome in adult sickle cell disease: a preliminary report. Crit Care 
Med. Mar. 2019;47(3):e263. https://doi.org/10.1097/CCM. 0000000000003628 .

12. Kuo K, Cornell T, Shanley T, Odetola F, Annich G. The use of extracorporeal membrane oxygenation in pediatric patients with sickle cell disease. Perfusion. Sep. 2013;28(5):424-32. https://doi. org/10.1177/0267659113485873.

13. Langer T, et al. Awake' extracorporeal membrane oxygenation (ECMO): pathophysiology, technical considerations, and clinical pioneering. Crit Care. Jun. 2016;20(1):150. https://doi.org/10. 1186/s13054-016-1329-y.

14. Mohite PN, et al. Extracorporeal life support in 'awake' patients as a bridge to lung transplant. Thorac Cardiovasc Surg.
Dec. 2015;63(8):699-705. https://doi.org/10.1055/s-00351546429.

15. Tudorache I. Lung transplantation for severe pulmonary hypertension-awake extracorporeal membrane oxygenation for postoperative left ventricular remodelling. Transplantation. Feb. 2015;99(2):451-8.

Publisher's Note Springer Nature remains neutral with regard to jurisdictional claims in published maps and institutional affiliations. 